On Oct. 12th, he was readmitted, and the splint removed : the limb is in a perfect line, but there is still a projection on the inner side. It seems as firm as a rock; but to be on the safe side, Mr. Partridge ordered another splint to be put on (plaster of Paris).

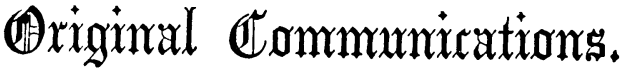

\section{CHRONIC INFLAMMATION OF THE ARTICULAR EXTREMITIES OF THE BONES.} By Thomas Bryant, F.R.C.S., Assistant Surgeon and Surgical
Registrar to Guy's Hospital.

[Read before the Medical Society of London, Oetober 18.]

UNDER the name of chronic inflammation of the articular extremities of the bones, I would venture to call attention to a large class of interesting diseases, which in their origin are seated exclusively in the bones, but which from their proximity to the joint, are almost always followed by disease of the articulation, unless arrested in an early stage.

It has been described by authors under different names, and is now generally known by the term strumous or scrofulous disease of a joint; and although I am disposed to doubt the wisdom of such terms, as applied to an inflammatory process, although that process may be frequently associated with strumous diathesis, I propose, with some hesitation, as a sub. stitute, the title of my present paper.

If there is one rule more important than another, and which we, as pathologists, should always strive to follow, it is to describe pathological changes by distinct terms, which are under stood by all, and to name diseases according to their pathological characters. And without entering at the present time upon the question, whether tubercular changes are not mere modified forms of the inflammatory process, I cannot for one moment doubt that the majority of the cases which are described by surgeons as strumous or scrofulous disease of a joint, and of the articular extremities of the bones, depend upon a chronic intlammation in the bone. The disease is, in its origin and progress, inflammatory; and by early treatment may be arrested. The pathological conditions found upon examination are those which an intlammatory process will produce, and it is quite exceptional to find in any bone that yellow cheesy material which pathologists so well know as strumous deposit; I do not deny that such a deposit is occasionally present, but the cases in which it is found are so rare, that we may fairly regard such a specimen as a pathological curiosity.

If, then, we confine the term strumous disease of a bone, as I believe we should, to such instances only where such a deposit is present, as surgeons, we shall seldom have occasion to employ it.

There are diseases of the synovial membrane which are included in the name strumous or scrofulous disease of a joint; but as my present communication is confined to such a disease when commencing in the bones, it is not my intention to allude further to them.

If we examine the articular extremity of a bone affected with this so called strumous disease, what shall we find?

In the earliest condition simple vascularity will be the principal morbid appearance, the cancelli containing more serum than natural; we shall soon, as the disease advances, observe that the articular extremity of the bone is larger than natural, this enlargement in some cases being very great. Upon making a section of the bone, the saw will break through its structure more easily than in a healthy specimen, its earthy constituents having diminished.

Upon comparing the structure of the diseased bone with a healthy specimen, it will be observed that its cancelli are much enlarged, the columns radiating from the shaft in a palm-like fashion, as if they had been spread out from downward pres. sure. These cancellated portions of the bone will appear more vascular than natural, and the cancelli will probably contain some pinkish serum.

If the inflammatory process is of a tolerably healthy character, parts of the bone will appear denser and more indurated than the remainder, evidently from the organisation of the inflammatory deposits, this deposit in bone always becoming osseous; too frequently, however, suppuration and death of the bone is the result, and a small or large sequestrum will be seen.

If we examine the denser portions of the bone forming its shell, it will be observed that it is thinner than natural ; that it also appears as if it had been dilated; where the articular extremity joins the shaft some new bone may be detected, thrown out as it were to support its dilated ends.

The articulating surfaces will, in some parts, appear more vascular than normal; in other cases, more advanced, portions will be found loose, and lying in the joint, having been like a slough thrown off. The denser portions of bone dying more rapidly than the cancellated.

If the cartilages are examined in the earliest stage of the disease, they may be found quite healthy; but when the disease has progressed so far as to involve the articular surfaces of the bone, the granular degeneration of their structure will be detected by the microscope, commencing upon the surface connected with the bone.

As the disease progresses, and when the surface of the bone is evidently injected and inflamed, the cartilage will be found to have been thrown off, and to be lying loosely upon the bone; and in more advanced or more chronic cases it will entirely have disappeared. The disease in the articular surfaces of the bone, and in the cartilage in apposition with it, progress at an equal rate, disease in one being certain to be followed by degeneration of the other, for the cartilage is as it were a dependent of the bone; for, receiving its nourishment through it, it must necessarily degenerate when that supply is interfered with, or cut off.

The disense having commenced in the bones, and as a consequence having involved the articular cartilages, which have undergone degeneration and decay, the synovial membrane generally becomes inflamed; the symptoms being very acute when any portion of the bone has died, and been discharged into the joint. In this case acute inflammation and suppuration are sure to follow, and rapid disorganisation of the joint is the result.

The description just given, forms the brief history of the pathological changes which may be witnessed in what is generally called strumous disease of a joint; it may take months, or even years, before the final destruction of the joint takes place; but unless arrested, such a termination must sooner or later be observed. The disease is evidently inflammatory in its origin and in its progress, although that process is of a low type. Occasionally, but very rarely, a genuine strumous deposit may be detected in the bone; the progress of the disease, however, is unaltered, it advances in the same slow but certain manner, and terminates as surely in disintegration of the joint; the strumous deposit indeed rendering such a termination more certain, as it acts, as it were, as a foreign body, and having always a tendency to degenerate and break down, the inflammation is kept up, until an outlet for its discharge has been found. This outlet is almost always through the joint; it may take place occasionally in other parts, but such an opening is very rare, for the disease has a constant tendency to progress jointwards, and whether it is associated with strumous deposit, or necrosed bone, these foreign bodies are, as a rule, discharged into the joint itself.

When the disease, as just described, takes place in the smaller bones, such as the carpal and tarsal, the same changes may be detected, and these changes are followed by the same results; the bones. may be found soft, and vascular, the cancelli being filled with bloody serum. Some will be seen pale on section, and perhaps denser from the organisation of the inflammatory products; in others the bone will be found dead; if confined to the cancellated structure it will be called carious, and if to the denser portion necrosed; and in these smaller bones such a result is very frequent. The cartilages will be also found to have undergone the same changes as in other joints, and the joints as a consequence will have advanced to different stages of disease.

Diagnosis. Having given thus briefly the pathological changes which may be seen in the disease we are now considering, I will proceed to the consideration of the symptoms by which such changes can be diagnosed; and it is important that the earliest conditions of this serious malady should be well understood, for it is only in an early step that much hope can be entertained of arresting its progress.

Commencing in the bones, it is to them that we must first look for any definite symptoms, and the earliest which will generally call our attention to the part, is some slight pain or aching; it will almost always be found in children, and those of a delicate and strumous habit; if the joint is one which can 
be easily examined, but little if any difference can be detected between it and its fellow; the pain is seldom great, but it is more of an aching character, and by firm pressure over some portion of the bone it will generally be increased.

As the disease advances, an evident enlargement of the bone will be detected, its articular extremity will appear as if dilated, and by comparison and measurement with its opposite will be variably enlarged; the enlargement in some cases being to the extent of inches. Some slight stiffness will then be observed in walking, and the pain will generally be increased by exercise. The cellular tissue external to the joint may now perhaps become involved, but only to the extent of some slight thickening. As the disease advances, and the cartilages become degenerate, the pain in the joint becomes greater, this pain being much increased by walking, or movement of the joint; startings of the limbs will now show themselves, and some spot about the joint will generally be more painful than another. When these symptoms are observed, effusion into the synovial cavity, if not present, will soon make its appearance, with all the symptoms of acute or chronic synovitis. The joint will then appear much enlarged and swollen, the points of bone being lost in the general enlargement; movement and pressure increase pain; and constitutional symptoms, hitherto very slight, now become much aggravated. Suppuration will sooner or later appear, and abscesses will open in all parts of the joint; and the character of the disease will be well declared in the total destruction of the articulation.

If this disease commences in the hip-joint, the early symptoms are seldom observed or witnessed, and limping is too often the first which attracts the attention of the parent, accompanied with some flexion and adduction of the thigh. There may be pain in the joint itself, and in the knee, or in the latter alone, and this will be generally aggravated by move. ment. When these symptoms are present, the disease will have generally advanced so far as to make the case one of a very serious nature, and it is then a difficult task to restore the joint to its former perfect condition.

In delicate strumous children the earliest complaint of pain or aching of a joint should not be disregarded, and if it is at all lasting, the earliest stage of this chronic inflammatory condition of the bone should be suspected, and at any rate the case should be treated as if the disease existed. Parents are apt to treat the complaints of children, particularly of joints, as "growing pains;" but I think, as surgeons, we should condemn such a practice, and although careful not to make the public fearful, and always on the look out for disease, we should alwars impress upon them the necessity of watehing for early symptoms, in order to seek advice for disease in its earliest conditions; when this disease has advanced far in the hip, the symptoms are too well marked to render it necessary for me to recall attention to them.

Treatment. There are two principal facts which we must always remember in the treatment of these cases; first, that the disease is inflammatory in its origin, and in its progress; and secondly, that it is almost always found in subjects of a weakly constitution, and of small power. The constitutional treatment becomes then an important consideration, and the local must be subjected to more general considerations.

The first point is to correct, if possible, the disposition to disease, to supply power to the patient to resist the morbid inflammatory process, and to overcome its effect. Tonics become then both valuable and necessary, in any form that may suit the patient. Iron, in any of its forms, is generally to be preferred, my favourite preparations are the iodide in the form of syrup, or the phosphate, the latter combined with the phosphate of lime. Cod-liver oil is a good tonic, particularly when little nourishment can be taken, and may also be given either with or without the iron, but it is better to give them together. The diet should be simple and nutritious, and alteratives occasionally given. The patient should have as much air as possible of a bracing character, and, in fact, all general considerations should be employed to improve the health and weakly powers.

The local treatment is important, although somewhat secondary to the constitutional; for however correct the former may be, it will be useless unless associated with the latter, and vice versâ.

The joint should be preserved absolutely at rest. If it is in the lower extremity, no pressure upon it by standing or walk ing should be allowed upon any consideration, and an elevated position is to be preferred. Blisters, setons, and such like applications are not to be employed, as they are seldom of any benefit, and in some cases do harm. Leeching is, at times, a valuable adjuvant; when local pain and heat are great, a few leeches over the part will often afford great relief; and warm or cold applications, according to the relief they confer, may be employed to allay pain.

Absolute rest, and moderate support by the application of strapping, either alone or with some mercurial ointment, as the compound mercurial is a valuable local remedy in more advanced cases.

In the earliest condition, when perhaps what are calledgrowing pains are the only local symptoms, it may be asserted that mere rest and consitutional means are generally sufficient to restore the patient and arrest the progress of the disease.

When the disease is still recent, but characterised by distinct symptoms, a mild mercurial, as the bichloride of mercury in small doses, or the grey powder, combined with tonies, should be judiciously employed; and under its use, together with such general means, as rest, and constitutional remedies, a speedy cure may often be obtained. Mercury in any other form may be pronounced as being positively injurious, except as an occasional purgative.

When it has advanced a stage beyond, and some decided symptoms of disease of the joint are present, the same treatment, with addition of the mercurial, as previously alluded to, and such local remeries as leeches, or hot or cold applications, may be all that is required. But when the disease has advanced still further, and the cellular tissue, etc., is partially involved, the mercurial ointment and moderate pressure by strapping may be advantageously employed; and if successful, the joint may be restored to its natural condition, or, at any rate, may be preserved as an useful limb.

But if the disease has progressed beyond this point, other considerations come into play; all hope of a restoration having passed away, it must be the aim and effort of the surgeon to make the best of a joint, which, as such, may be now considered as destroyed.

When the cartilages have degenerated or disappeared, and the synovial membrane and cellular tissue have become involved, the object of the surgeon must be to obtain an anchylosis of the joint.

Constitutional means, as already given, must still be sedulously employed, and perfect rest, and immobility of the joint preserved by splints, or strapping in the position which is most to be desired. By such treatment carefully applied, and after the lapse of long periods of time, such a result may generally be obtained; but it can only be by great care and much patience, both on the part of the surgeon and his patient.

When the presence of some necrosed bone may be suspected, or diagnosed, and perfect disorganisation of the joint has taken place, some operative interference may become necessary. If the part observed should be the shoulder or elbow, there is no doubt that excision of the joint, or of the diseased portion, is the right operation to be performed; as perfect success may here generally be expected, and partial is even better than the loss of the limb.

When the hip and knee-joints are the diseased parts, it is impossible to speak of the operation of excision in the same positive and unqualified terms. The success which has followed the operation in some cases speaks undoubtedly in strong language in its favour; but there is an opposite side, and it cannot be doubted that such success is not so general. When it is possible to diagnose the existence of some necrosed bone, the joint being, as a result, already disorganised; and if the surgeon is tolerably certain about being able to remove it, - the operation of excision of the diseased portion is certainly the correct treatment, if other general considerations are not opposed to such a practice. But, in the hip-joint, such a diagnosis is seldom possible; and the removal of a portion only is quite useless, for the part will heal up partly, but again to open, and be followed by all the symptoms which previously had existed. And, although it is not my wish to enter, upon the present occasion, upon the subject of excision of joints, I may state that I firmly believe the operation, when applied to the upper extremity, as the shoulder and elbow joints, to be a most valuable one; and that, where there is a choice, it should be preferred, as a rule, to amputation. But when the same practice is to be applied to the hip, knee, and ankle joints, $I$ cannot speak in the same positive manner; and although there may be cases, as experience has already proved, in which the operation has been followed by success, and therefore there may be others in which such a practice may be adopted, still, as a rule, I do not believe the operation will hold ground, or be found nearly so valuable as its advocates would lead us to believe. Exceptional cases may occur, where the operation may 
be selected; but the rule, I believe, must be in the opposite direction. When the case is a good one for excision of the knee -that is, when the disease is chiefly confined to the articular facets of the joint, and where the powers of the patient are good-it may, I think, be forcibly asserted that there is generally a fair chance of obtaining an anchylosed limb, and that the operation should then never be performed. In other cases, when the disease is more extensive, involving a large portion of the articular extremities of the bones, and when the porvers of the patient are bad, the chances of success by excision become small.

There may be some intermediate cases, where the disease is in the heads of bones, and where that disease may be removed, where the chances of recovery, if left to return, are slight (for she may be unable to throw off or get rid of the necrosed or carious bone), and where the powers of the patient are tolerably good: in such, if the surgeon can remove the bone which is keeping up by its pressure the disease in the joint, and is thus preventing its recovery-in such, the operation may be pronounced of value; but, in others, amputation is the right operation. And, although no surgeon should remove a limb without serious con sideration and strong reasons, still it must be borne in mind that the aim should be not only to save it, but to make it useful. There is no doubt that limbs are now saved more frequently than of old by means of excision, but there is also no doubt that many of them are useless, if not absolutely in jurious and in the way; and, although I would not wish you to believe that I am at all an opponent to what is called conservative surgery, still mere conservatism must be injurious, if it is not associated with practical tendencies; and, unless a limb can be restored sufficiently to enable its owner to pursue his duties or his occupation, its absence would be less injurious, and amputation had better be performed at once.

In the hip-joint, the same general rules are applicable. When the disease is confined to the surface of the articulation, and the bones are still in their natural position, surely no such operative interference as excision can be required. When the head of the femur and the acetabulum are both extensively diseased, and when by operative interference only a portion of the dead bone can be removed, but small hope can be entertained of recovery; and the only cases in which removal of the head of the femur appears advisable are those in which the bone is dis. located upon the dorsum, and necrotic, and which by its presence keeps up profuse suppuration and constitutional irritation; but such cases can hardly be regarded as excision of a joint, but may be classified more naturally with the operations for caries or necrosis.

I have now thus briefly called your attention to the pathology, diagnosis, and treatment of what has been generally described as strumous disease of a joint; and have, I trust, proved to you, as briefly as possible, that the disease is essentially an inflammatory one, and that it should, therefore, more correctly be denominated chronic inflammation of the articular extremity of the bones. I might have quoted numerous cases to illustrate the different points brought forward, from my notes of two thousand cases of injuries and necrosis of the joints, which I have now in my possession; but I feel sure that the experience of those surgeons who are present will supply the deficiency. I have given, as briefly as possible, the results of inquiries which I have been pursuing for many years; and if I have been able to place a large and serious class of cases in their proper position in our surgical pathology, my end will have been obtained.

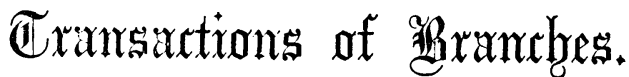

\section{EAST YORK AND NORTH LINCOLN BRANCH. ON POINTS OF ELECTION IN AMPUTATION OF THE LEG. \\ By Kelburne King, M.D., Hull. [Read September 23rd.]}

Ox the 29th of May, 1858, I met Dr. Beckett of Hull, in consultation regarding $\mathrm{C}$. $\mathrm{T}$., aged 40 , a schoolmaster, who gave the following account of himself.

On December 1st, 1835, he was shipwrecked in the Gulf of St. Lawrence, and suffered very great hardship, being for several days exposed to extreme cold, with very insufficient clothing. Indeed, of a ship's company of fourteen men, only six survived, and these were all more or less disabled. He suffered frost-bite of both his feet; and the whole soft parts of the soles, and the metatarsal and phalangeal hones of both fect, sloughed away: the remaining bones were gradually covered over, partly by the remaining skin, partly by a cicatrix, though there was always a tendency to ulceration, more particularly in the left foot. He for many years experienced but little trouble, except what resulted from lameness. By having his foot well padded with worsted stockings, he contrived to walk about with the aid of a stick. Complete cicatrisation, however, never took place in the left foot, from which there always was more or less discharge, particularly from a spot on the upper and outer side. Things continued in this state till about five years ago, when he observed a small warty-looking substance, about the size of a pea, springing out from the ulcerated spots before referred to. This from the first was exceedingly sensitive, and it increased in size very slowly and gradually for some years. About two years ago, it began to grow more rapidly, and, for some time before I saw him, it had occasioned severe and constant pain.

On examining the left foot, I found a stump, consisting of the proximal ends of the three inner metatarsal and of the cuboid bones, covered with skin on the dorsal aspect; but the natural texture of the sole of the foot was destroyed, and its place supplied by a thin integument adherent throughout to the bones. The elastic part of the heel and sole was quite gone, and the lower part and sides of the calcaneum were as distinctly marked almost as in the skeleton, being covered only by this thin integument. On the upper and outer part of the foot, and springing apparently from the labial bone, was a large warty tumour, with a deep fissure on its under surface, and about the size of a hen's egr. The skin in the neighbourhood also had a roughened warty appearance, as if about to take on the same action. This tumour was exquisitively sensitive to the touch, and was the seat of continual acute lancinating pain, preventing him from obtaining sleep, and rendering his life miserable. A probe, forced into the fissure, entered the substance of the cuboid bone, which was felt soft and carious. He was much debilitated. He had a sallow unhealthy aspect, and was of a nervous, irritable temperament. His sufferings were such that he was willing to submit to anything to obtain relief.

$\mathrm{He}$ had had a great variety of treatment, regular and irregular; and, among other remedies, had used strong caustic applications, but had never obtained the slightest relief.

It was apparent, then, that the removal of this malignant and painful tumour was necessary for this man's comfort, and even for his life: and we resolved to effect it by the knife, as the most effectual and satisfactory method. It was obvious that what remained of the foot must be sacrificed, and the state of the integuments prevented the performance of Mr. Syme's operation at the ankle-joint; so that it was necessary to amputate through the bones of the leg. The skin around the malleoli was perfectly sound and healthy; and I proposed to make a cutaneous flap, principally from the inner ankle, and to divide the bones with the saw, about an inch above the articulation. A very long stump would thus be left, and one which could not be expected to bear any weight-an objection which would have been of great importance when it was supposed that the weight of the body must rest on the bent knee, unless the patient were in a position to pay a large sum for an artificial limb. But since it has been known that a simple and inexpensive instrument can be made, by means of which the weight of the body is borne on the projecting heads of the tibia and fibula; the stump, whether long or short, being secured in a wooden box, and the power of extension and flexion of the leg upon the thigh being preserved,-this objection loses all its force. This man was not in a favourable condition to undergo a very serious operation, such as that of amputation at what is termed the point of election, where the wound would have been larger, the part of the body removed greater, and the medullary cavity of the bones would have been necessarily opened.

On my making this proposal to Dr. Beckett, he at first referred me to the following passage from Mr. Fergusson's work on Practical Surgery: "Where the surgeon has a choice of parts, he should prefer operating about the middle of the leg." (p. 351, 1st edition.) But, on my pointing out some facts which I shall refer to afterwards, he sanctioned my proposal; and accordingly, on June 10th, we performed the following operation. The man being placed under the influence of chloroform, a semilunar incision was made over the inner ankle, commencing at the tendo Achillis, and terminating at the centre of the auterior aspect of the leg. This incision was 